\title{
Turning Segways into Robust Human-Scale Dynamically Balanced Soccer Robots
}

\author{
Jeremy Searock, Brett Browning, and Manuela Veloso \\ School of Computer Science, The Robotics Institute, \\ Carnegie Mellon University, Pittsburgh, PA, 15213 \\ j.searock@andrew. cmu.edu \\ \{brettb, veloso\}@cs.cmu.edu
}

\begin{abstract}
The Segway Human Transport (HT) is a one person dynamically self-balancing transportation vehicle. The Segway Robot Mobility Platform (RMP) is a modification of the HT capable of being commanded by a computer for autonomous operation. With these platforms, we propose a new domain for human-robot coordination through a competitive game: Segway Soccer. The players include robots (RMPs) and humans (riding HTs). The rules of the game are a combination of soccer and Ultimate Frisbee rules. In this paper, we provide three contributions. First, we describe our proposed Segway Soccer domain. Second, we examine the capabilities and limitations of the Segway and the mechanical systems necessary to create a robot Segway Soccer Player. Third, we provide a detailed analysis of several ball manipulation/kicking systems and the implementation results of the CM-RMP pneumatic ball manipulation system.
\end{abstract}

\section{Introduction}

Considerable research has been conducted involving human-robot interaction [8], and multi-robot teams [9, 10, 11]. With the inception of RoboCup robot soccer [7], multiagent team coordination within an adversarial environment has been studied extensively. But, the dual topic of human-robot coordination in an adversarial environment has yet to be investigated. This research involves the intelligent coordination of mixed teams of humans and robots competing in adversarial tasks against one another. The results of this research will further the technology necessary to allow both humans and robots to productively work together in complex environments requiring real time responses.

In order to further investigate human-robot interaction in team tasks, we have developed a new game called Segway soccer. The rules of the game are a combination of soccer and Ultimate Frisbee with an emphasis on fostering humanrobot interaction. In order to investigate different perception and cognitive abilities, the humans and the robots need be placed on an equal physical level by utilizing the Segway Human Transporter (HT) and the Segway Robot Mobility Platform (RMP). Several mechanical systems are needed to equip the RMP with the physical abilities necessary to allow a game. We present the Segway and the domain of Segway Soccer 
in Section 2. We explain the modifications necessary to create a Robot Segway Soccer Player in Section 3 along with the mathematical models necessary to choose among the different ball manipulation systems in Section 4. The implementation details and experimental results of the CM-RMP Robot Soccer Player are in Section 5 , and our conclusions and future plans in Section 6.

\section{Segway Soccer Platform and Game}

The Segway ${ }^{\mathrm{TM}}$, developed by Dean Kamen, is a two-wheeled dynamically balanced mobility platform. The Segway has onboard sensors and computer controllers that continually and independently command each wheel in order to maintain balance. The human rider controls the velocity of the Segway by leaning forward, shifting the center of mass, and causing the Segway to drive forward in order to rebalance.

The RMP has provided a robust robotic agent on which to create human-scale robots. The Segway RMP is a 'roboticized' Segway HT consisting of three main modifications. First, a CAN Bus interface is exposed to enable two way, high speed electronic communication with the platform. Second, the Segway's control software is modified to enable a computer to send direct velocity commands to the platform. The third change involves attaching a large mass of about $23 \mathrm{~kg}$ at a height of $50 \mathrm{~cm}$ from the robot wheel base. This serves the purpose of raising the robot's center of gravity which slows down the RMP's falling rate in order to allow the control loop to operate effectively.

Using the Segway platform, we have developed a new game called Segway Soccer, which consists of teams of humans and robots competing in a game where the rules are a combination of soccer and Ultimate Frisbee ${ }^{1}$. The objective of the game is to score the most goals by kicking an orange size 5 soccer ball into a goal which is $2.5 \mathrm{~m}$ wide. One key contribution from ultimate Frisbee is that once a player is declared to have possession of the ball by a referee, the player cannot dribble. Instead, the player has a $1 \mathrm{~m}$ radius in which to reposition and pass to a teammate. This rule is in place for safety reasons, so that robots and humans will not contest each other for possession. Furthermore, to ensure robots and humans will collectively be involved, a mixed team cannot officially score unless both a robot and a human interact with the ball on the way to the goal.

Placing humans, robots, and robot competitors on an equal physical level using the Segway platform allows their different perception and cognitive abilities to be tested.

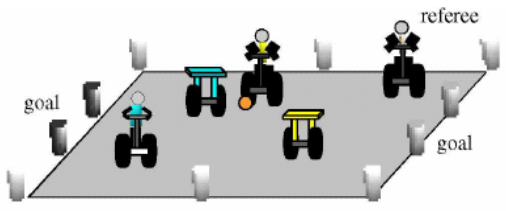

Fig. 1. Segway Soccer field

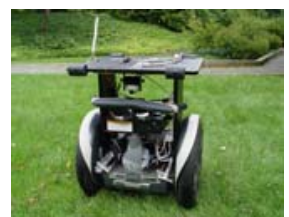

Fig. 2. Segway RMP

\footnotetext{
${ }^{1}$ Ultimate Players Association, [http://www.upa.org]
} 
The dynamic balancing, speed, and size of the Segway allows this human-robot interaction at a human scale. The Segways can travel at $3.5 \mathrm{~m} / \mathrm{s}$ and have a footprint of $48 \mathrm{~cm}$ by $64 \mathrm{~cm}$. Our Segway RMP also has its camera mounted $1.5 \mathrm{~m}$ above the ground, providing a human height perspective.

\section{Turning the Segway RMP into a Soccer Player}

This new domain of human-robot interaction raises the requirements of robot mechanical systems to a more sophisticated level. The challenge becomes designing adequate hardware that will allow a robot to safely and robustly operate in an outdoor environment along with humans in a competitive soccer game.

In meeting this challenge, we have developed 4 key goals. First, the soccer player must be autonomous by perceiving the world, making decisions, and acting without human intervention. Second, the player must be able to interact with human players by recognizing their presence and communicating. Third, the player must be able to manipulate a ball well enough to be competitive with humans. Lastly, safety must be considered in every aspect to prevent injury to humans and damage to equipment.

With these goals in mind, we have to consider the many challenges that accompany designing and implementing a complete robotic system. Cost effectiveness, processing power, perception, weight distribution, and resistance to shock are all important considerations. The unique motion of the Segway also introduces problems not seen with other platforms.

The Segway moves forward by tilting over and driving the wheels in order to rebalance. This motion can lead to the ball becoming stuck underneath the body and wheels of the Segway. This causes the wheels to lose contact or traction with the ground making the Segway unable to sufficiently maintain balance. Any consequential fall could potentially damage equipment. As a solution, guards, consisting of modified rubber mud flaps, were placed in front of the wheels and computer control software was implemented to prevent the RMP from interacting with the ball unless it knows the manipulation system can kick.

Another challenge introduced with Segway Soccer is that there is no unique playing surface; it can be played on grass, Astroturf, or cement. Changes in grass height, ground softness, and surface texture alter the dynamics necessary to manipulate the ball. Unlike other robotic soccer platforms, the Segway also tips up to +/- 20 degrees with respect to the vertical; thus, any attached kicking plate and system will also tip. This requires the manipulation system to be robust enough to manipulate the ball under changing conditions.

With a basic infrastructure in place, we added two laptop computers to interpret the world and control the RMP. One laptop is used to provide the RMP with the capability to process data from a pan/tilt CCD camera and another laptop to quickly decide what action to take and send commands to control the actions of the RMP. Speakers were added to allow the robot to speak to its teammate to help control the flow of the game.

Finally, hardware must be able to protect the components of the Segway from damage during a fall. The laptop computers and ball manipulation system components are mounted as close as possible to the bottom of the Segway reducing their falling 
distance and the shock they will absorb. The laptops are also securely fastened with straps preventing them from being ejected from the confines of the RMP body. Steel safety stands were added to reduce the total distance the Segway will fall once it is no longer capable of dynamically balancing. The stands mount onto the side of the RMP and only allow it to fall over 30 degrees from the vertical.

\section{Ball Manipulation Systems}

One of the main challenges to using a Segway RMP to play soccer lies in designing a ball manipulation system that allows a Segway platform to kick a ball to the scale of an outdoor human game. The need for passing in the game and the inability of the robot to safely propel the ball by running into it necessitates the development of a kicking mechanism. Although kickers of all forms have been developed in RoboCup, there have been no formalized comparisons of different mechanisms. We present an analysis of the common kicking mechanisms as well as a detailed implementation and evaluation of a pneumatic kicker as a step towards developing a scientific based method for selecting kicking mechanisms.

\subsection{Kicking System Design Considerations}

A ball manipulation system can be described as a mechanical manipulator used to accelerate a ball to a desired velocity in a desired trajectory. This can be achieved in many different ways with various actuators. The most common systems come from the realm of robot soccer as seen in RoboCup [7] competitions. These include pneumatic, spring, solenoid, rack and pinion, and rotating plate systems. A careful analysis of the following factors is needed to determine which kicking system best fits a given platform and environment:

- Speed-How fast should the ball be kicked?

- Accuracy- What distribution is acceptable? Should it only kick straight?

- Kick capacity- How many kicks are possible or needed in a game?

- Response time-How long will it take for a signal to result in a kick?

- Recovery time- How long before a second kick is possible?

- Safety- Is the system likely to injure a human player or bystander?

- Complexity- What is the build time? What parts are required?

- Weight-Can the robot carry the payload?

- Size- Will the system fit on the robot and perform adequately?

- Price- Is it worth the extra money?

- Power-Can the robot carry the power supply? How long will the supply last?

- Reliability/Maintenance-How likely will parts break under normal competitive play? How expensive and complex is the maintenance?

- Transportability- Can the system be transported in an airplane? Are spare parts readily available? ${ }^{2}$

\footnotetext{
${ }^{2}$ The latter is especially critical given the international nature of RoboCup competition.
} 
With these considerations in mind, the actuating system must be chosen. For each option, we present the basic system components, the mathematical models necessary to properly specify an appropriate actuator, and an example comparing each option to the pneumatic system we implemented and describe in section (5).

\subsection{Spring Loaded Mechanisms}

Spring kicking mechanisms use an extension or compression spring(s) to store and then release energy to propel the ball. As such, a mechanism is needed to tension the spring(s) and a trigger to release the stored energy. Such mechanisms must be robust, and are non-trivial to design. Apart from the obvious complexity, spring strength is coupled to kicking power, but a more powerful spring is more difficult to retract and hold. This relationship leads to potential problems during a soccer game where the time to reload a powerful spring can take several seconds if a cheaper less powerful motor is used. Springs do provide the best power density out of the given the options $[4,5]$.

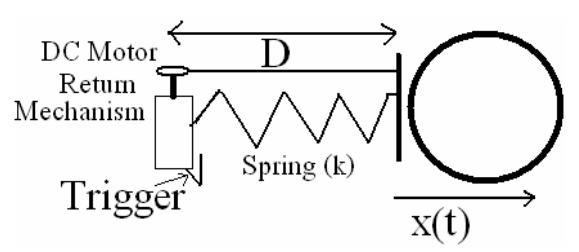

Fig. 3. Spring Kicking Mechanism Schematic

For a spring with a spring constant of $k$, a kick length of $D$, and a kicking mass of $m$, the force equation and resulting equations of motion for the spring mechanism are:

$$
\begin{gathered}
\ddot{x}=-k x \cdot m^{-1} \quad x(0)=D \quad \dot{x}(0)=0 . \\
x(t)=D \cos (\sqrt{k / m} t) .
\end{gathered}
$$

For the Segway, the pneumatic kicking system model predicts the ball will be kicked at a $4.3 \mathrm{~m} / \mathrm{s}$ max velocity. Using the above equations, one can determine the spring constant, $k$, necessary to achieve a similar speed with a similar stroke length to the pneumatic model $(0.1524 \mathrm{~m})$. Assuming a kicking mass of $0.85 \mathrm{~kg}$, a spring constant of $676 \mathrm{~N} / \mathrm{m}$ would be necessary. This would require a force of at least $103 \mathrm{~N}$ in order to load and hold the spring at $0.1524 \mathrm{~m}$ for a kick. Depending on motor size and consequentially cost, the spring would take one to several seconds to reload for another kick. Complexity, reload time, and cost are the liming factors for the spring kicker design.

\subsection{Rotating Plate Mechanisms}

Rotating plate kickers consist of two or more flat surfaces, bars, or other contacts arranged in a balanced paddle boat configuration. [1] The shaft of the paddle wheel is connected to a DC motor. The angular velocity of the paddle wheel determines the end velocity of the ball, although there is great variability due to the potential 
variation in the contact point. Pulse Width Modulation can be used to vary the speed of the wheel and thus vary the power of the kick. Rotating plate mechanisms require a significant amount of space to mount the paddle wheel and the drive motor. Furthermore, for larger robots, rotating plates become extremely dangerous to human operators. A rotating plate mechanism scaled to the size of a Segway would have to be approximately $18 \mathrm{~cm}$ by $38 \mathrm{~cm}$. The plates would be rotating fast enough and with enough power to cause injury to humans who happen to fall off of their HT into a kicking device. As a result, we do not consider a rotating plate mechanism in depth.
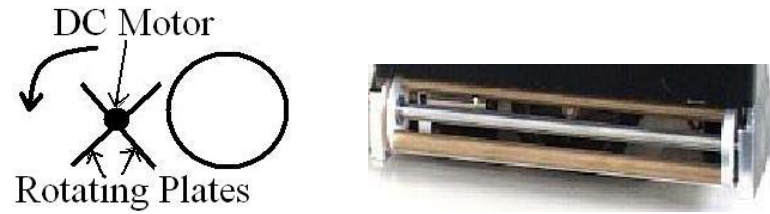

Fig. 4. Rotating Plate Kicker Schematic and Physical Example

\subsection{Rack and Pinion Systems}

Rack and pinion systems are driven by DC motors and thus the ball velocity is dependent on the output power of the motor. For a rack and pinion motor system with a back emf of $k_{e}$, voltage of $V$, forward torque per amp of $K$, terminal resistance of $\mathrm{R}$, pinion radius of $r$, gear ratio of $N$, and total kicking components mass of $m$, the following are the equations of motion:

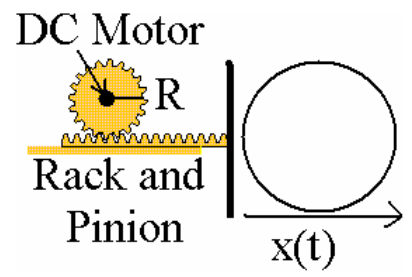

Fig. 5. Rack and Pinion Kicking System Schematic

$$
\begin{gathered}
\ddot{x}=\frac{K}{m r^{2} R}\left(V r-k_{e} \dot{x}\right) \quad x(0)=0 \quad \dot{x}(0)=0 . \\
x(t)=\frac{V r}{k_{e}} t-\frac{m r^{3} R V}{N K k_{e}^{2}}\left(1-e^{-\frac{k_{e} K N}{m r^{2} R} t}\right) .
\end{gathered}
$$

A rack and pinion system with a powerful motor is comparable to the other options but the price and design requirements are more significant. Using an 80W Maxon 
motor [12], a $0.015 \mathrm{~m}$ pinion radius, $1: 1$ gear ratio, and a total kicking mass of $1.3 \mathrm{~kg}$, the rack and pinion system can accelerate the ball to a theoretical velocity of $6.7 \mathrm{~m} / \mathrm{s}$ in $0.1524 \mathrm{~m}$ (6in). With a motor efficiency around $75 \%$ and the friction forces acting against the sliding rack, the actual velocity will be closer to $3.5 \mathrm{~m} / \mathrm{s}$.

The Segway does not have enough space to implement a single rack and pinion system. Two rack and pinions would be needed since one rack and pinion could not be placed in the middle of the Segway due to the handle bar mounting. This would require two motors or a much larger single motor to actuate both rack and pinions. This requirement makes this system unfeasible for use on a Segway platform. The two high power motors would also be costly [3].

\subsection{Solenoid Systems}

A solenoid kicker consists of a solenoid that creates a magnetic field around a shaft that is propelled by the field and accelerated away from the solenoid. The shaft is returned by a built in return spring. Consider a solenoid kicking system with a current of $I$, ampere turns of $N$, plunger radius of $r$, a return spring constant of $k$, and a total kicking mass of $m$. The differential equation of motion is given in equation (5). Since this equation can only be solved by numerical means, we approximate the result here for analysis purposes by treating the solenoid force as being constant for the duration. The results are given by equations (6) and (7).

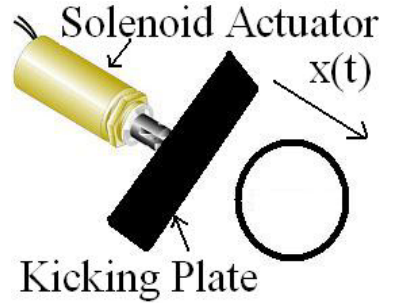

Fig. 6. Solenoid Kicker Schematic

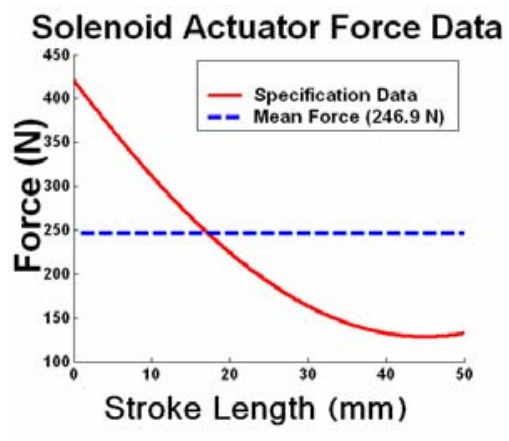

Fig. 7. Solenoid Force v. Stroke and Approximate Force

$$
\begin{gathered}
\ddot{x}=m^{-1}\left(1 / 2 \mu_{0} \pi(r N I / x)^{2}-k x\right), x(0)=0 \quad \dot{x}(0)=0 . \\
\ddot{x}=\mathrm{m}^{-1}\left(\mathrm{~F}_{\mathrm{avg}}-k x\right), \quad x(0)=0 \quad \dot{x}(0)=0 . \\
x(t)=F_{\text {avg }} / k(1-\cos (\sqrt{k / m} t)) .
\end{gathered}
$$

Most commonly available solenoids produce approximately $400 \mathrm{~N}$ of force and generally have small stroke lengths on the order of $0.0254 \mathrm{~m}$ (1in), which limit its ability to effectively contact a ball. For the solenoid shown in figure (7) [13], 
assuming a return spring constant $k$ of $99 \mathrm{~N} / \mathrm{m}$, and a kicking mass of $1.5 \mathrm{~kg}$, a ball would be kicked at $4.1 \mathrm{~m} / \mathrm{s}$ over a $0.0508 \mathrm{~m}$ stroke length (2in). With the effects of friction and motor efficiency the actual speed would be closer to $3 \mathrm{~m} / \mathrm{s}$. This is comparable to the pneumatic system but the smaller stroke length limits is ability to effectively manipulate a ball during a game. The high voltage requirement may also raise safety issues.

\subsection{Pneumatic Systems}

Pneumatic piston systems usually consist of one or two actuating cylinders, an air reservoir, solenoid valves to control the air flow, a source of compressed gas in the form of compressed air or liquid carbon dioxide $\left(\mathrm{CO}_{2}\right)$, and a regulator to maintain a specified pressure. The decision between $\mathrm{CO}_{2}$ and compressed air depends on the availability of $\mathrm{CO}_{2}$. Air compressors normally only operate up to 150 psi while $\mathrm{CO}_{2}$ tanks fill to several thousand psi. This higher pressure allows the cylinders to be fired with a higher output force resulting in a stronger kick. The higher pressure also significantly increases the kick capacity of the system. The major drawback of $\mathrm{CO}_{2}$ is that it is not easily transportable or available in foreign locations. Additionally, its rapid expansion during each kick results in thermal issues such as the formation of condensation near electronic parts. As a result, a compressed air approach is often used instead.

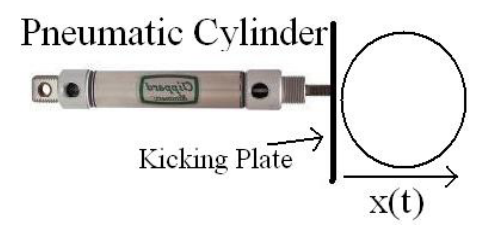

Fig. 8. Pneumatic Kicker Schematic

The pneumatic cylinder pistons connect to a kicking plate that contacts the ball. The plate can vary in material and shape dependent upon application. The one or more pistons can be fired at the same time or in a synchronized order to achieve a directional kick. A pneumatic system offers a wide range of options in its configuration and employment. For a pneumatic system with power factor, $f$, combined kicking mass, $m$, return spring constant, $k$, and operating at a pressure, $P$, the equations of motion are:

$$
\begin{gathered}
\ddot{x}=m^{-1}(f P-k x), \quad x(0)=0 \quad \dot{x}(0)=0 . \\
x(t)=f P \cdot k^{-1}(1-\cos (\sqrt{k / m} t)) .
\end{gathered}
$$

For a Segway, a suitable cylinder would be approximately $0.254 \mathrm{~m}$ (10in) long, $0.01905 \mathrm{~m}$ in diameter and produce $274 \mathrm{~N}$ for force at $140 \mathrm{psi}$. The pistons are the 
only moving parts and the air tank consumes the most space. The price of a pneumatic system is also fairly cheap. A functional system can be bought for less than $\$ 150$. The air used to power the cylinders is naturally accessible and can be refilled quickly during a soccer game with an onboard air compressor. The system has a low chance of malfunctioning and becoming inoperable during a game because the only moving parts are the cylinder shafts. $[2,8]$

\section{Implementation and Results}

With the considerations presented, we chose to use a pneumatic approach due to its relative simplicity, low cost, and transportability. Figure (9) shows the resulting arrangement

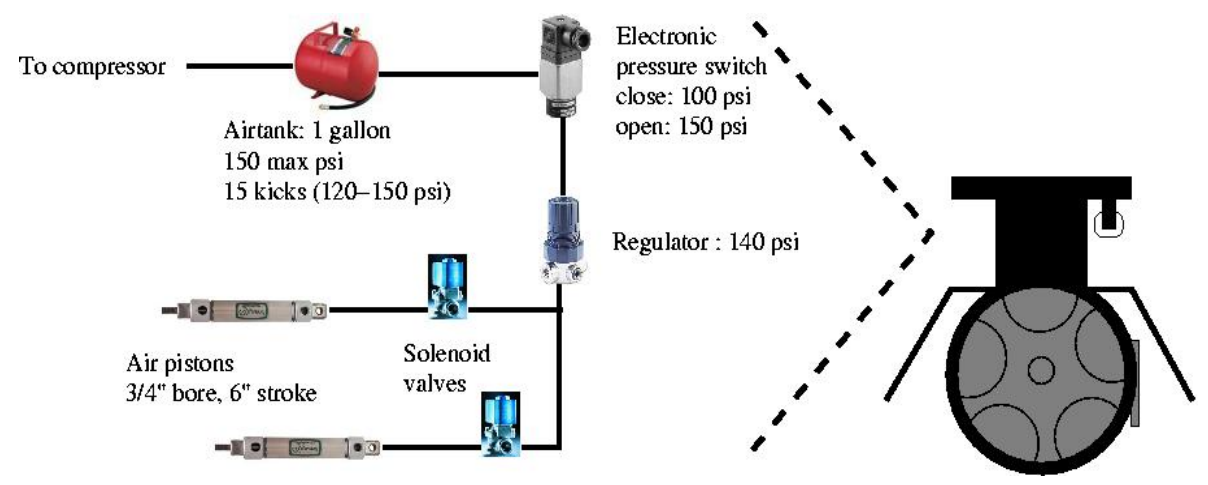

Fig. 9. Schematic diagram of the implemented CM-RMP pneumatic kicking system

Two $0.01905 \mathrm{~m}$ bore, dual acting pneumatic cylinders were chosen as the main actuating components. This size cylinder provides adequate power with a sturdy shaft that can sustain unexpected stress. Dual acting cylinders do not have a return spring to reset the cylinder shafts back to their original position. This allowed us to implement a return mechanism with just enough force to reset the kicking plate without significantly affecting the output force. We used 4 x No.64 (3.5in x 1/4in) rubber bands to return the kicking plate. Two cylinders also allow for directional kicking.

\subsection{Air Reservoir Options}

The air reservoir can be designed in two different ways. The reservoir can be large enough to hold enough kicks for the entire game or an onboard air compressor can refill a smaller reservoir. If the robot has enough room to house a larger tank, not having an air compressor allows the overall system to be simpler. We used a 1 gallon tank, which provides a sufficient number of kicks as seen in figure (10). We have an onboard compressor that turns on after 15 kicks and shuts off when the tank pressure reaches 150 psi. The compressor is controlled by a microcontroller that also monitors 
a mechanical pressure switch that opens at 150 psi. As a result, the operation of the compressor is completely automated.

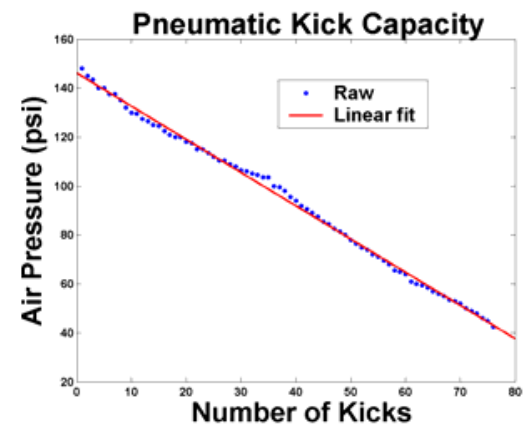

Fig. 10. Number of Kicks v. Reservoir Pressure

\subsection{Velocity Test Results}

The cylinders accelerate the ball to a max velocity of approximately $3.5 \mathrm{~m} / \mathrm{s}$, which is sufficient for a two on two game of Segway soccer. The velocity can increase to 4.5 $\mathrm{m} / \mathrm{s}$ if the Segway RMP is moving at the ball when it kicks it. The theoretically predicted top speed for a stationary kick is approximately $4.3 \mathrm{~m} / \mathrm{s}$. The loss in velocity is due to the efficiency of the pneumatic cylinders, an imperfect impact with

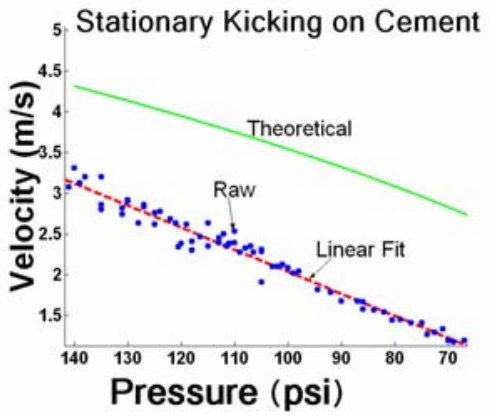

Fig. 11. Experimental and Theoretical Pressure v. Kick Velocity

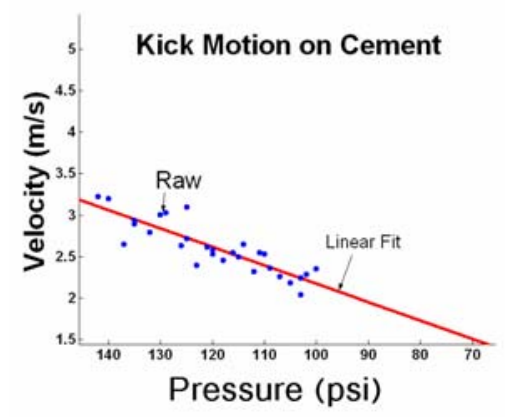

Fig. 12. Pressure v. Kick Motion Velocity

the ball, and ground friction. An experiment was setup using one of the cylinders, a small kicking plate, and a golf ball. The velocity of the golf ball was measured on a cement floor. This experiment was designed to significantly lower the effects of impact and friction losses. Through these tests, it was determined that the pneumatic cylinder alone had an efficiency of $75 \%$. These losses are due to several factors including cylinder friction, exiting air resistance, and flow rate limitations. Impact 
losses and ground friction account for an additional $2 \%$ loss. The theoretical and experimental kick speed versus cylinder pressure plot is shown in figure (11). Furthermore experiments were conducted measuring the speed of the ball when the Segway RMP played back a kick motion in which it swung its base forward and simultaneously kicked. These results are seen in figure (12).

\subsection{Accuracy}

The kick is sufficiently accurate as seen by the distribution in figure (13). The mean is $122 \mathrm{~mm}$ and the standard deviation is $175 \mathrm{~mm}$. The mean error can be mostly accounted for by experimental error in lining up the kick. In practice, this mean and variance will be modified by the robots ability to position itself next to the ball.

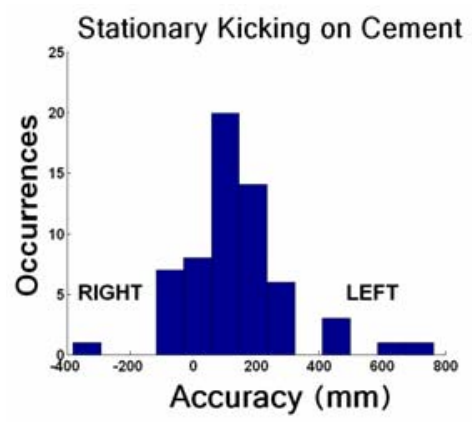

Fig. 13. Histogram of Stationary Kicking on a Cement Surface

\section{Conclusions and Future Work}

We have presented a new domain, Segway Soccer, in which to investigate human-robot interaction within adversarial environments. Within this domain we have identified the challenges behind placing humans and robots on the same physical level utilizing the Segway platform. We have also established a scientific basis on which to choose a ball manipulation/kicking system for any size robot soccer player and have outlined the other mechanical systems necessary to make a Segway RMP physically capable of playing Segway Soccer along with humans. With our analysis, we have accurately implemented a pneumatic ball manipulation system, which robustly kicks the ball fast enough and with enough accuracy to make passing and goal scoring possible.

Our future work will focus on further developing the concept of Segway Soccer and on additional mechanisms to increase the soccer playing abilities of the Segway, such as recovering the Segway from a fallen state.

\section{Acknowledgements}

Thank you to Mike Sokolsky and David Rozner for their help in the construction of the mechanical devices. Finally, we would like to thank the funding sources, who 
supported and guided this research and development to address this specific problem. This research was sponsored by the United States Army under Grant No. DABT6399-1-0013. The views and conclusions contained in this document are those of the authors and should not be interpreted as necessarily representing official policies or endorsements, either expressed or implied, of DARPA, the US Army, or the US Government.

\section{References}

1. 1.S. Behnke et. al., "Using Hierarchical Dynamical Systems to Control Reactive Behavior." RoboCup-99:Robot Soccer World Cup III. Berlin: Springer, 2000, p.189

2. M. Ferraresso et. al., "Collaborative Emergent Actions Between Real Soccer Robots." RoboCup-2000:Robot Soccer World Cup IV. Berlin: Springer, 2001, p.297

3. Ng Beng Kiat et. al., "LuckyStar II-Team Description Paper." RoboCup-2000:Robot Soccer World Cup IV. Berlin: Springer, 2001, p.543

4. G. Wyeth et. al., "UQ RoboRoos: Achieving Power and Agility in a Small Size Robot." RoboCup-2001:Robot Soccer World Cup V. Berlin: Springer, 2002, p.605

5. R. Cassinis et. al., "Design for a Robocup Goalkeeper." RoboCup-99:Robot Soccer World Cup III. Berlin: Springer, 2000, p.255

6. A. Bredenfeld et. al., "GMD-Robotst." RoboCup-2001:Robot Soccer World Cup V. Berlin: Springer, 2002, pp. 648-9

7. M. Asada et. al. "An overview of RoboCup-2002 Fukuoka/Busan". AI Magazine, 24(2): pages 21-40, Spring 2003

8. M. Nicolescu, M. J Mataric, "Learning and Interacting in Human-Robot Domains", Special Issue of IEEE Transactions on Systems, Man, and Cybernetics, Part A: Systems and Humans , Vol. 31, No. 5, pages 419-430, C. C. White and K. Dautenhahn (Eds.), 2001

9. M.B. Dias and A. Stentz. "Opportunistic Optimization for Market-Based Multirobot Control". Proceedings of the IEEE/RSJ International Conference on Intelligent Robots and Systems IROS 2002, 2002

10. M. Ferraresso, et al., "Collaborative Emergent Actions Between Real Soccer Robots." RoboCup-2000:Robot Soccer World Cup IV. Berlin: Springer, 2001, pp.297-300

11. N. Kiat, Q. Ming, T. Hock, Y. Yee, and S. Yoh, "LuckyStar II-Team Description Paper." RoboCup-2000:Robot Soccer World Cup IV. Berlin: Springer, 2001, pp. 543-546

12. Maxon Motors, "F 2260, Graphite Brushes, 80 Watt, No.880," http://www.mpm. maxonmotor.com] P. 95

13. Solenoid City, "Push Type Tubular Solenoid, Series S-70-300-H," [http://www. solenoidcity.com] 